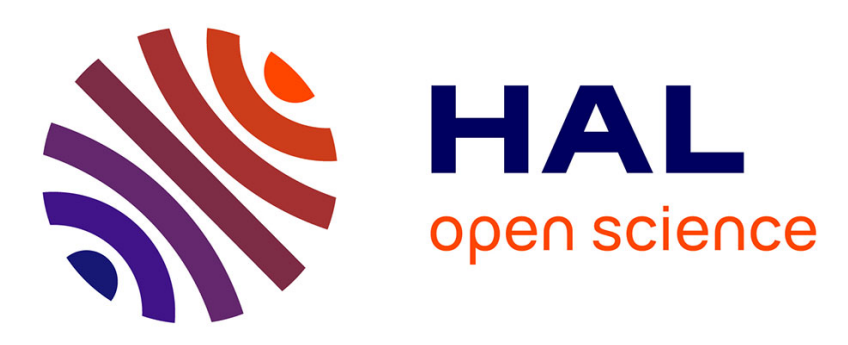

\title{
Core-clock genes Period 1 and 2 regulate visual cascade and cell cycle components during mouse eye development
}

Udita Bagchi, Shumet Gegnaw, Nemanja Milićević, Cristina Sandu, Jacoline ten Brink, Aldo Jongejan, David Hicks, Perry Moerland, Marie-Paule Felder-Schmittbuhl, Arthur Bergen

\section{To cite this version:}

Udita Bagchi, Shumet Gegnaw, Nemanja Milićević, Cristina Sandu, Jacoline ten Brink, et al.. Coreclock genes Period 1 and 2 regulate visual cascade and cell cycle components during mouse eye development. Biochimica et Biophysica Acta - Gene Regulatory Mechanisms , 2020, 1863 (10), pp.194623. 10.1016/j.bbagrm.2020.194623 . hal-03447260

\section{HAL Id: hal-03447260 \\ https://hal.science/hal-03447260}

Submitted on 24 Nov 2021

HAL is a multi-disciplinary open access archive for the deposit and dissemination of scientific research documents, whether they are published or not. The documents may come from teaching and research institutions in France or abroad, or from public or private research centers.
L'archive ouverte pluridisciplinaire HAL, est destinée au dépôt et à la diffusion de documents scientifiques de niveau recherche, publiés ou non, émanant des établissements d'enseignement et de recherche français ou étrangers, des laboratoires publics ou privés. 


\section{Core-clock genes Period 1 and 2 regulate visual cascade and cell cycle components during mouse eye development}

Udita Bagchi ${ }^{a, c}$, Shumet Gegnaw ${ }^{a, c}$, Nemanja Milićevića,c, Cristina Sandua, Jacoline B. ten Brink ${ }^{c}$, Aldo Jongejan $^{b}$, David Hicks ${ }^{a}$, Perry D. Moerland ${ }^{b}$, Marie-Paule Felder-Schmittbuhla ${ }^{*}$, Arthur A. Bergen ${ }^{c, d, e}$

${ }^{a}$ Centre National de la Recherche Scientifique, Université de Strasbourg, Institut des Neurosciences Cellulaires et Intégratives, F-67000 Strasbourg, France

bagchi@inci-cnrs.unistra.fr (U.B : ORCID 0000-0001-7726-0319), gegnaw@inci-cnrs.unistra.fr (S.G), sandu@inci-cnrs.unistra.fr (C.S), davidhicks@inci-cnrs.unistra.fr (D.H), feldermp@inci-cnrs.unistra.fr (M.P.F.S)

${ }^{b}$ Amsterdam UMC, University of Amsterdam, Bioinformatics Laboratory, Department of Clinical Epidemiology, Biostatistics and Bioinformatics, Amsterdam Public Health research institute, Meibergdreef 9, Amsterdam, the Netherlands (NL)

a.jongejan@amsterdamumc.nl (A.J), p.d.moerland@amsterdamumc.nl (P.D.M)

'Department of Clinical Genetics, AMC, Meibergdreef 9, 1105 AZ Amsterdam, NL

j.b.tenbrink@amc.uva.nl (J.B), n.milicevic@amc.uva.nl (N.M : ORCID 0000-0002-8062-7270), aabergen@amc.uva.nl (A.A.B)

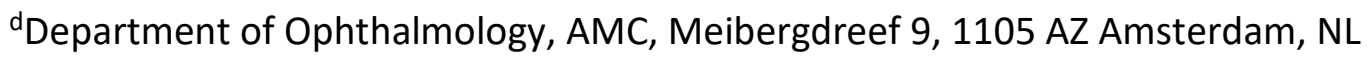

'The Netherlands Institute for Neuroscience (NIN-KNAW), Royal Netherlands Academy of Arts and Sciences, Meibergdreef 47, Amsterdam, NL

*Correspondance : Dr. Marie-Paule Felder-Schmittbuhl (ORCID 0000-0003-3539-1243), Institut des Neurosciences Cellulaires et Intégratives (INCI), CNRS UPR3212, 8 Allée du Général Rouvillois, 67000, Strasbourg, France. Telephone: +33-0-388456644. Email address: feldermp@inci-cnrs.unistra.fr 


\begin{abstract}
The retinas from Period 1 (Per1) and Period 2 (Per2) double-mutant mice (Per1 /-Per2 ${ }^{\text {Brdm } 1)}$ display abnormal blue-cone distribution associated with a reduction in cone opsin mRNA and protein levels, up to 1 year of age. To reveal the molecular mechanisms by which Per1 and Per2 control retina development, we analyzed genome-wide gene expression differences between wild-type (WT) and Per1 - ${ }^{-}$Per2 ${ }^{\text {Brdm1 }}$ mice across ocular developmental stages (E15, E18 and P3). All clock genes displayed changes in transcript levels along with normal eye development. RNA-Seq data show major gene expression changes between WT and mutant eyes, with the number of differentially expressed genes (DEG) increasing with developmental age. Functional annotation of the genes showed that the most significant changes in expression levels in mutant mice involve molecular pathways relating to circadian rhythm signaling at E15 and E18. At P3, the visual cascade and the cell cycle were respectively higher and lower expressed compared to WT eyes. Overall, our study provides new insights into signaling pathways -phototransduction and cell cycle- controlled by the circadian clock in the eye during development.
\end{abstract}

Keywords: circadian clock, eye, photoreceptor, differentiation, transcriptomics

\footnotetext{
${ }^{1}$ Abbreviations

CPM, counts per million; DEG, differentially expressed genes; FDR, false discovery rate; IPA, Ingenuity pathway analysis; LD, light-dark cycle; PCA, principal component analysis; qPCR, quantitative polymerase chain reaction; SCN, suprachiasmatic nucleus; WT, wild-type; ZT, zeitgeber time.
} 


\section{Introduction}

The coherent functioning of the circadian clock, driven by genetic and endogenous factors as well as environmental cues, is pivotal in orchestrating the rhythmicity of biological functions. The circadian system operates through a hierarchical system of oscillators. These oscillators occur in several peripheral tissues and brain areas, with the suprachiasmatic nucleus $(\mathrm{SCN})$ of the hypothalamus being the primary pacemaker or the master clock [1]. The molecular clockwork machinery comprises transcription factors encoded by "clock genes", which interact in interlocked transcriptional/translational regulatory loops. The key positive loop encompasses CLOCK and BMAL1 heterodimers. The negative feedback loop is formed by the Period (PER) 1-3 and Cryptochrome (CRY) 1-2 proteins which inhibit the transcriptional activity of BMAL1/CLOCK dimer. An important interconnecting secondary loop of the circadian oscillator involves both the nuclear receptor families of REV-ERBs and RORs (respectively named after the anti-sense Rev-Erbo in relation to the c-ErbA- $\alpha$ /Thra gene; encoded by Nr1d1-2 and Retinoic acid-related Orphan Receptors, encoded by Rora-c). In addition, the core clock network has many other putative regulatory genes, such as $D b p, H I f$, Tef, Ciart, Csnk1ع, Csnk18, Fbxl3, Fbxl21, Nfil3, Bhlhe40, Bhlhe41, and Arnt/2 [2].

It is well known that the peripheral tissue oscillators are synchronized by outputs from the SCN, resulting in coordinated rhythmic oscillations of peripheral organs. Without the driving force of the SCN, these organs run out of phase $[3,4]$. The capacity to synthetize melatonin in a rhythmic manner, in vitro, suggested the idea of an endogenous clock in the eye, located in the retina [5, reviewed in 6]. Directly sensitive to the light/dark cycle, the retina is responsible for the synchronization of circadian rhythms generated within the SCN $[7,8]$. Several functions in the mammalian eye are controlled by the retinal circadian clock (reviewed in $[9,10])$ extending from the expression of photopigments $[11,12]$ to visual sensitivity [13-15]. Retina specific functions such as photoreceptor outer segment disk shedding and RPE mediated phagocytosis as well as susceptibility to phototoxicity have been linked to the clock [16-18]. In addition, the rate of mitosis measured in the cornea $[19,20]$ along with the daily production of aqueous humor in the ciliary body contributing to intraocular pressure [21, 22], are also well-studied rhythmic cellular processes.

The circadian system is known to time the early development of the mouse visual cortex [23]. It is during these developmental stages that circadian rhythms optimize growth and neurobehavioral development [2426]. Protein products of clock genes regulate cellular processes within the wild-type eye during development, at least until adulthood [15, 27-29]. Loss of Rev-Erba (Nr1d1) expression results not only in several defects during retinal visual processing, but also of retinal sensitivity to ambient light [30,31]. We previously 
reported a number of developmental anatomical/histological defects related to eye physiology up to 1 year of age in the Per1 ${ }^{-/}$Per $2^{\text {Brdm1 }}$ mutant mice $[32,33]$. These defects include a reduced number of blue cone opsin (Opn1sw) expressing cells, decreased steady-state levels of blue cone opsin gene expression and delayed differentiation of rod inner and outer segments [34]. Sawant and coworkers [35] recently showed that the circadian clock gene Bmal1 is implicated in spatial patterning of cone opsins in the retina. This corroborates the hypothesis that circadian clock genes are essential for proper cone development. Finally, in Cry1/Cry2 double-mutant animals, cone function is also compromised [36]. Nonetheless, the detailed molecular mechanisms linking clock genes and cone development remain to be elucidated.

Understanding how the circadian clock controls retinal development is essential to apprehend how its disturbance could lead to pathogenicity [37]. The recent development of whole genome-wide transcriptomic approaches allowed systematic analysis of temporal gene expression and identification of clock target genes in specific cell-types/organs $[15,38]$. It was shown for example that rhythmic regulation of gene expression programs relies on BMAL1/CLOCK binding to DNA that primes target genes for tissue specific transcription activation [39, 40]. Furthermore, transcriptome characterization in 12 mouse organs over time revealed $43 \%$ of protein-encoding genes possessing a rhythmic expression profile [41]. Recently, the first transcriptome atlas of a diurnal non-human primate in more than 60 tissues/organs also unraveled a wide array of rhythmic tissue-specific genes, extending the proportion of protein encoding genes regulated by the clock in at least one tissue to about $80 \%$ [42]. To date, transcriptomic analysis in ocular tissues has provided an extensive list of cyclic processes in various compartments of the eye [15, 42]. Yet, the understanding of developmental signaling pathways potentially under the control of clock gene expression is still sparse. Hence, the aim of the present study is to understand transcriptional difference in the timing of developmental processes and the functional implications that arise due to clock perturbations.

We currently present the developmental expression profiles of clock transcripts in mouse WT eyes between embryonic day 13 (E13) and postnatal day 24 (P24). Furthermore, we have characterized genomewide differential gene expression in the whole eye of Per1 ${ }^{-/}$Per2 ${ }^{B r d m 1}$ mutant versus wild-type at E15, E18 and P3 and identified functional categories and pathways. We found that the Per1/Per2 ablation significantly affects gene expression of the secondary regulatory loop members of the circadian clockwork, of the phototransduction pathway as well as of cell cycle components during development. 


\section{Materials and methods}

\subsection{Animal Care}

Mice were handled according to the European Parliament and The Council of the European Union Directive (2010/63/EU). All experimental procedures conformed to the Association for Research in Vision and Ophthalmology Statement on Use of Animals in Ophthalmic and Vision Research. The homozygote double mutant mice Per1 $1^{-/-}$Per2 ${ }^{\text {Brdm1 }}$ carrying the loss-of-function mutation of Per1 and Per2 genes [32, 33] were obtained as a generous gift from Dr. U. Albrecht (University of Fribourg, Switzerland). Wild-type (WT) and mutant animals (both on mixed C57BL/6J/129Sv background) were housed and bred in the Chronobiotron animal facility (UMS 3415, CNRS-University of Strasbourg) on a light-dark (LD) cycle (12 h light/12 h dark, 300 lux during the light phase, dim red light $<5$ lux during the dark period), with an ambient temperature of $22 \pm 1{ }^{\circ} \mathrm{C}$. Animals were supplied with standard chow diet and water ad libitum. Control and mutant mice were age-matched, and mostly male mice were used in the post-natal groups.

\subsection{Genotyping}

Genotyping was performed by PCR amplification on mouse-tail DNA separately for the WT and KO alleles. The primer sequences were as follows: Per1 KO fwd: 5'-ACAAACTCACAGAGCCCATCC-3' and Per1 KO rev: 5'-ACTTCCATTTGTCACGTCCTGCAC-3'; Per2 mutant fwd: 5'-TTtGTTCTGTGAGCTC CTGAACGC-3' and Per2 mutant rev: 5'-ACTTCCATTTGTCACGTCCTGCAC-3'; [32, 33]; Per1 WT fwd: 5'GTCTTGGTCTCATTCTAGGACACC-3' and Per1 WT rev: 5'-AACATGAGAGCTTCCAGTCCTCTC-3'; Per2 WT fwd: 5'-AGTAGGTCGTCTTCTTTATGCCCC-3' and Per2 WT rev: 5'-CTCTGCTTTCAACTCCTGTGTCTG-3'. PCR conditions comprised of $35 \mathrm{cycles}$ of $30 \mathrm{sec}$ at $94^{\circ} \mathrm{C}, 30 \mathrm{sec}$ at $56^{\circ} \mathrm{C}$ for Per1 (WT and KO) or $58^{\circ} \mathrm{C}$ for Per2 (WT and mutant), $1 \mathrm{~min}$ at $72{ }^{\circ} \mathrm{C}$ followed by a final step for 5 min at $72{ }^{\circ} \mathrm{C}$.

\subsection{Eye Sampling}

Both wild-type and the mutant mice were subjected to timed breeding. After confirmation of the presence of a vaginal plug at E0, female mice were immediately transferred to a separate cage until the appropriate embryonic $(E)$ and postnatal $(P)$ developmental stages were reached. qRT-PCR analysis was performed for E13, E15, E18, P0, P3, P13 and P24 only in WT samples. The comparative transcriptome study was performed only on samples from E15, E18 and P3 from WT and mutant animals, based on the periods of genesis of cones and rods in mice (respectively around E13-E16 and E18-P3 $[43,44])$. The E15, E18 and P3 WT samples used for RNA sequencing were distinct from those used for qPCR. For very young mice, 
decapitation was used. Cervical dislocation was performed only for older animals. Eye sampling was performed at the Zeitgeber time (ZT4), $4 \mathrm{~h}$ after lights were switched on. A Zeitgeber is any external or environmental cue that entrains/synchronizes an organism's biological rhythms (in our case it would be only light). Whole eye globes were enucleated, snap-frozen on dry-ice and stored at $-80^{\circ} \mathrm{C}$ until use. Histological validation was performed on whole eyes sampled on P0 and P3 animals from same breeders and showed no obvious difference between genotypes (Supplementary Figure S1).

\subsection{RNA extraction and quality control}

Mouse whole eyes were individually homogenized in $500 \mu \mathrm{l}$ TRI Reagent (Molecular Research Center, Cincinnati, $\mathrm{OH}, \mathrm{USA}$ ) and incubated for $5 \mathrm{~min}$ at room temperature. $100 \mu \mathrm{l}$ of chloroform were added to each lysate and after 2 min incubation at room temperature the mixture was centrifuged using phase-lock gel tubes (Heavy, 2ml; QuantaBio, Beverly, MA, USA) at $12000 \times \mathrm{g}$ for $15 \mathrm{~min}$ at $4{ }^{\circ} \mathrm{C}$. Following the phase separation, the RNA from the upper aqueous phase was precipitated with equal volume of $70 \%$ ethanol and purified using the RNeasy micro kit (Qiagen $\mathrm{GmbH}$, Hilden, Germany) according to manufacturer's protocol, including the DNase digestion step. The RNA was eluted with $14 \mu$ of RNase-free water. RNA concentration and purity were measured using NanoDrop ND-1000V 3.5 Spectrophotometer (NanoDrop

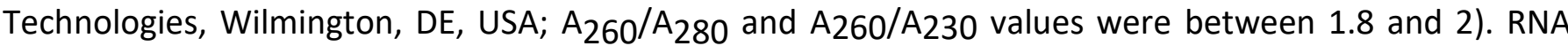
quality was evaluated with the Bioanalyzer 2100 with RNA 6000 Nano Kit (Agilent Technologies, Santa Clara, CA, USA; RNA integrity numbers were 9.7-10).

\subsection{Real-time quantitative RT-PCR (qRT-PCR)}

Total RNA $(1 \mu \mathrm{g})$ was used to synthetize cDNA using the High-Capacity RNA to cDNA Kit (Thermofisher, Courtaboeuf, France). qPCR was performed to analyze gene expression along development at E13, E15, E18, P0, P3, P13 and P24 using the 7300 Real Time PCR system (Thermofisher) and the hydrolysed probebased TaqMan chemistry as previously described in [45]. Serial dilutions created from the pool of all cDNA samples were used to calculate the amplification efficiency for each assay (values were between 1.8 and 2 for all assays). Each PCR reaction was done in duplicate. Nine circadian clock genes were examined: Arntl, Clock, Per1, Per2, Per3, Cry1, Cry2, Nr1d1, and Rorb (Supplementary Table S1). The PCR program was: 10 min at $95{ }^{\circ} \mathrm{C}$, followed by 40 cycles of denaturation at $95{ }^{\circ} \mathrm{C}$ for $15 \mathrm{sec}$ and annealing-elongation at $60{ }^{\circ} \mathrm{C}$ for $1 \mathrm{~min}$. The PCR conditions were 1 x TaqMan Universal PCR Master Mix, No AMPErase UNG (Thermofisher); $1 \times$ Gene Expression Assays mix (containing forward and reverse primers and cognate probe; Thermofisher); and $1 \mu \mathrm{l}$ of cDNA in a total volume of $20 \mu \mathrm{l}$. At the end of the elongation step, 
fluorescence data acquisition was performed using the 7300 System Sequence Detection Software V1.3.1 (Thermofisher) and qBase software (free v1.3.5; [46]) was used for data analysis. Transcript levels were normalized to Gapdh and Sdha [47]. Data were submitted to Shapiro-Wilk normality test (data for Per1 and Nr1d1 did not pass). Differences among the age groups were analyzed using one-way ANOVA followed by Holm-Sidak post-hoc test or (when data did not pass the normality test) a non-parametric KruskallWallis test followed by a Dunn's post hoc test (Sigmaplot v12, Systat Software, Inc.). The post hoc analysis is detailed in Supplementary Table S2.

\subsection{Construction of RNA-seq Libraries and RNA sequencing}

RNA-Seq libraries were constructed from 500 ng of total RNA (E15, E18 and P3) using the KAPA mRNA HyperPrep Library Preparation Kit 005302-11-1 (Roche Sequencing Solutions, Pleasanton, CA USA) for Illumina Platform HiSeq 4000 as per manufacturer's instructions. RNA was treated with magnetic oligo-dT beads (Lot No: 005328-9-1) to capture the poly (A) RNA and was chemically fragmented into a desired size (around 200-300 bp) using heat in the presence of $\mathrm{Mg}^{2+}$. These RNA fragments were used for first and second strand cDNA synthesis. (A) tailing was added to the blunt ends of the dscDNA to enable adapter ligation with the $(T)$ base overhangs. These adapter-ligated library DNA were purified and used for enrichment by using adapter-specific PCR. The libraries were amplified using a mixture of KAPA HiFi HotStart RdyMix (2X) and Lib. Amp. Primer Mix (10X) (Roche Sequencing Solutions, Pleasanton, CA USA) to produce strand-specific PCR products. The library amplification process was verified using flash gel visualization along with cleanup steps. By using Agilent Bioanalyzer, quality and size distribution of the cDNA library was checked. Fragments size for the cDNA library were between 200 and 500 bp, with a peak at $\sim 300$ bp. Qubit 2.0 Fluorometer (Life Technologies, Foster City, CA, USA) was used for the quantification of libraries. The cDNA library was sequenced by single-end sequencing of 50 bases length on Illumina Hiseq 4000 sequencer (Illumina, San Diego, CA, USA).

\subsection{RNA sequencing data analysis}

Reads were subjected to quality control (FastQC v.0.11.5, dupRadar [48], Picard Tools) showing low duplication- and appropriate gene detection rates, trimmed using Trimmomatic v0.32 [49] and aligned to the reference mm10 mouse genome (Ensembl GRCm38 v87) using HISAT2 (v2.0.4) [50]. Counts were obtained using HTSeq (v0.6.1) [51] with parameters "-m union -f bam -r name -s no -a 10 -t exon -i gene_id" and the mouse GTF from Ensembl (version 87). 
Statistical analyses were performed using the edgeR [52] and limma [53] R/Bioconductor packages using R (v3.4.3) and Bioconductor (v3.6). 22,911 genes with more than 5 counts in at least 3 of the samples were retained. Count data were transformed to log2-counts per million (logCPM), normalized by applying the trimmed mean of M-values method [54] and precision weighted using voom [55]. Differential expression was assessed using an empirical Bayes moderated t-test within limma's linear model framework including the precision weights estimated by voom. Resulting $p$-values were corrected for multiple testing using the Benjamini-Hochberg false discovery rate. An adjusted $p$-value $\leq 0.05$ was considered significant, and an additional fold change cut-off $(\leq-1.5$ or $\geq 1.5)$ was applied to identify differentially expressed genes. Additional gene annotation was retrieved from Ensembl (release 91) using the biomaRt R/Bioconductor package. Principal component analysis (function plotPCA, package DESeq2) was performed on the logCPM values of the top-500 most variable genes.

Supplementary Figure S2a shows that the relative clock gene mRNA quantities in WT eye globes between E15, E18 and P3, as assessed by qRT-PCR (data from Figure 1), confirm the data obtained by RNA-Seq. In addition, qPCR analysis performed on whole eye globes from WT and Per1 ${ }^{-/}$Per2 ${ }^{\text {Brdm } 1}$ mice confirmed the gene expression changes between genotypes measured by RNA-Seq (Supplementary Fig S2b). These analyses agree with other studies $[56,57]$. The two series of quantifications (qRT-PCR/RNA-Seq) were obtained with distinct sample series, thus also providing a biological validation of our results.

Results from RNA-sequencing in Per1 ${ }^{-/}$Per2 ${ }^{\text {Brdm1 }}$ eye samples show the absence of reads in specific genomic regions from the Per1 and Per2 loci corresponding to the deletion mutations initially introduced in these genes [32, 33], thus validating the loss of expression of PER1 and PER2 (Supplementary Figure S3).

\subsection{Canonical Pathway Analysis}

Canonical pathway enrichment analysis was performed using IPA (Ingenuity) by using both up and down regulated genes in mutant vs WT (adjusted $p$-value $<0.05$ and fold change $\leq-1.5$ or $\geq 1.5$ ), at each age (Ingenuity Systems, version 24718999, accessed in November 2018). The list of all identified transcripts in our RNA-Seq dataset was used as a reference background. Significance of enrichment was calculated using a right-tailed Fisher's Exact Test and corrected for multiple testing using the Benjamini-Hochberg false discovery rate $(p<0.01)$. 


\section{Results}

\subsection{Clock gene expression in WT control mouse eyes during development}

We first performed a temporal analysis of core clock gene expression by quantitative RT-PCR (qRT-PCR) in developing whole eyes from WT mice. We chose seven age time points (E13, E15, E18, P0, P3, P13, P24) spanning photoreceptor genesis and maturation. Our data revealed distinct temporal gene expression dynamics for key core clock components (Bmal1 or Arntl, Clock, Per1, Per2, Per3, Cry1, Cry2, Nr1d1 and Rorb) during eye development (Figure 1 and Supplementary Table S2).

This initial analysis revealed that all clock genes examined are already expressed at E13. Using one-way ANOVA, their abundance showed a significant age-related change (Figure 1). The relative expression level of Bmal1 (Arntl) mRNA reached a peak just after birth at P3 while maintaining moderate expression at other ages (Figure 1a). Similarly, Rorb mRNA had significantly highest expression around P3 (Figure 1i). In contrast, a steady increase in gene expression was maintained by Clock gene from E13 until P3 (Figure 1b). Among Period genes, Per1 and Per2 expression peaked at birth (P0) whereas Per3 expression culminated only at the postnatal ages (P13 and P24; Figure 1c-e). Cry2 also reached the highest expression levels close to eye opening at P13 (Figure 1f). Finally, Cry1 mRNA had a peak in gene expression at birth (P0) (Figure 1e). Nr1d1 had the most striking profile, increasing expression levels between E13 and P24 with a foldchange difference of 19 , whereas differences between minimal and maximal expression did not reach 4fold for other clock genes (Figure 1h). This initial analysis helped us to select time points for further indepth whole-genome RNA-Seq analysis.

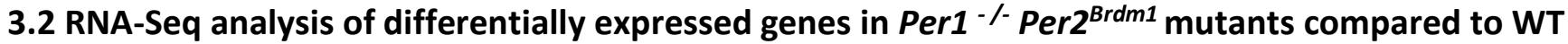 controls}

\subsubsection{Whole transcriptome changes}

We performed RNA sequencing to compare the whole eye gene expression profiles between Per1 ${ }^{-1-P e r} 2^{\text {Brdm1 }}$ and WT mice, as a function of time during embryonic (E15, E18) and postnatal (P3) ages. Principal component analysis clearly showed age and genotype effects in the whole eye transcriptome (Figure 2). Age-related effects were pronounced and segregated with the first principal component (PC). This explains most of the variance in the data (PC1: 56\%). Indeed, both in mutant and WT mice, many genes (respectively 14046 and 15445) were significantly differentially expressed (adjusted p-value <0.05) between different developmental stages. This possibly reflects the extensive gene expression changes occurring in eye development between E15 and P3. Genotype-related effects segregated on the second 
principal component, which explained only $7 \%$ of the variance in the data. Thus, gene expression profiles cluster together for mutants and WT at E15, whereas clear differences can be observed between mutant and WT expression profiles at E18 and P3.

\subsubsection{Specific transcriptome changes between genotypes}

Analysis of differentially expressed genes (DEG; adjusted $p$-value $<0.05$ and fold change $\leq-1.5$ or $\geq 1.5$ ) of the Per1 - Per $2^{\text {Brdm1 }}$ versus WT whole eyes showed an increasing number of DEG during development. At time points E15, E18 and P3, we respectively found 36, 200 and 367 genes with significantly increased expression. At the same time points, we observed 18, 90 and 273 genes with significantly decreased expression in the mutant mice (Figure 3a). Only limited numbers of genes (35 up and 30 down) are differentially expressed at more than one developmental stage.

Of the 23 genes most likely involved in the molecular clockwork [2], eight genes (Per3, Nr1d2, Ciart, Bhlhe41, Rorc, Tef, Npas2 and Dbp) displayed statistically significant changes between WT and mutants, in at least one age group (Figure 3b). In contrast, expression of Per3 and Nr1d2 showed a large increase in the mutant eyes at all ages. Ciart displayed specific increase at E18 in the mutant, whereas Bhlhe41, Rorc and Tef mRNAs were specifically increased at P3. Npas2 expression was markedly reduced and Dbp significantly upregulated in the E18 and P3 mutants.

All the differentially expressed genes at E15 within the cut-off range are presented in Figure 4a. Selected DEGs are highlighted in the volcano plots for E18 and P3 age groups (Figure 4b and c). Most of them relate to the eye and, more specifically, to the photoreceptor, to neuronal development and to the clock. The complete lists of DEGs sorted by statistical significance are listed in Supplementary Table S3 (E15 mutant versus WT), Table S4 (E18 mutant versus WT) and Table S5 (P3 mutant versus WT) respectively.

\subsection{Functional differences between gene expression profiles in the Per1 ${ }^{-/} \operatorname{Per}^{\text {Brdm } 1}$ and WT whole eyes}

To evaluate gene expression profiles on the level of canonical pathways, we performed enrichment analyses on the genes differentially expressed in the different age groups (adjusted $p$-value $<0.05$ and fold change $\leq-1.5$ or $\geq 1.5$ ) using the Ingenuity Pathway Analysis (IPA) software. We focused our analysis on the presence of canonical pathways, since these are the simplest molecular representations of the relevant biological events. 
Top canonical pathways associated with genes differentially expressed at E15 are related to 'circadian rhythm signaling' and the 'adipogenesis pathway' (Figure 5a). Quite expectedly, 'circadian rhythm signaling' is also the top-ranked canonical pathway at E18 followed by four other pathways suggesting enrichment in these signaling molecules (Figure 5b).

Ten canonical pathways were significantly enriched with genes differentially expressed between Per1 ${ }^{-1}$ Per2 ${ }^{\text {Brdm1 }}$ mutants and WT at the postnatal age P3 (Figure $5 \mathrm{c}$ ). The topmost significant canonical pathway was related to Phototransduction, which agrees with the aberrant phenotype (alteration of photoreceptor development at early postnatal age) previously found in the mutant [34].

The components of phototransduction cascade, which were mainly upregulated in the mutant, are highlighted in Figure 6a,b (see also Supplementary Figure S4 and S5a,c). We also found increased expression of photoreceptor-specific genes, like Prph2 (peripherin 2), Impg1 and Impg2 (interphotoreceptor matrix proteoglycan 1 and 2), Prcd (photoreceptor disc component), Rp1/1 (RP1 like 1), Nr2e3 (Nuclear Receptor Subfamily 2 Group E Member 3), Prom1 (prominin 1) and Mpp4 (membrane palmitoylated protein 4) (details in Supplementary Table S5), indicating the differentiation of the photoreceptor complex.

Interestingly, a substantial fraction of genes previously implicated in human congenital ocular pathologies were found with increased expression levels in P3 mutants: for instance Rpe65 (retinitis pigmentosa of the RPE65 type), Rp1 (retinitis pigmentosa 1), Pon1 (serum paraoxonase/arylesterase 1), Bbs7 (Bardet-Biedl syndrome 7), Nxn/1 and Nxn/2 (Rod-derived cone viability factors), Ptgds (prostaglandin D2 synthase), Rs1 (retinoschisis (X-linked, juvenile) 1), Samd7 (SAM domain-containing protein 7), Tacstd2 (tumor-associated calcium signal transducer 2), Cdhr1 (cadherin related family member 1), Efemp1 (EGF containing fibulin extracellular matrix protein 1), Crybb2 (crystallin, beta B2) and Mgp (Matrix Gla protein) (details in Supplementary Table S5).

The Per1 /- Per2 ${ }^{\text {Brdm1 }}$ mutants at P3 also displayed a decrease in expression of cell-cycle-associated genes (Figure $5 c$ and $7 a, b, c)$. There was a clear down-regulation of genes belonging to the 'Cell Cycle Control of Chromosomal Replication' pathway (Figure 7a). DNA replication licensing factors like $\mathrm{Mcm} 2, \mathrm{Mcm} 3, \mathrm{Mcm} 6$ and $C d t 1$, cell division cycle proteins like $C d c 6$ and $C d c 45$ along with essential enzymes like Pole and Top2a were the down-regulated components (Figure 7a). The 'Cyclins and Cell Cycle Regulation' pathway had significantly reduced expression levels of some of the major E2F family genes like E2f2, E2f7 and E2f8 along with the cyclins B1 (Ccnb1) and D1 (Ccnd1) (Figure 7b). Within these two enriched cell-cycle-related 
pathways, only cell cycle genes $C d k 18$ (cyclin-dependent kinase 18) and Ppp2r2b (protein phosphatase 2 regulatory subunit beta, a negative regulator of division) were found upregulated. It is known that there is a decrease in cell division at this period of normal eye development [58]. This is confirmed in our data by the major downregulation of genes belonging to the 'DNA replication' and 'Cell cycle' GO terms in WT eyes between E18 and P3 (Supplementary Figure S5d). However, downregulation of these genes appears even more prominent in the mutant eyes, based on the p-values of these canonical pathways (Supplementary Figure S5d).

\section{Discussion}

The circadian clock is a major regulator of physiology and behavior through the temporal control of gene expression programs on a $24 \mathrm{~h}$ scale. The molecular role and mechanism of action of clock genes in developmental processes have lately received increased interest [59-64]. Here we focus on the comparison between the eye transcriptome of Per1 ${ }^{\%}$ Per2 ${ }^{B r d m} 1$ and WT mice at E15, E18 and P3. In our dataset, we observed differential expression of clock genes, especially from the regulatory feedback loops. Most prominently at P3, mutant eyes display the most extensive alterations: significant upregulation of phototransduction-related genes, together with a reduction in cell cycle-related transcripts. Our work provides further evidence that clock genes play a role in eye development, likely by taking part in the signaling between differentiation programs and regulation of cell division.

Using immunohistochemistry, we previously described that retinas from Per $1^{-/}$Per2 ${ }^{\text {Brdm1 }}$ mutant mice display altered photoreceptor differentiation with reduced blue cone numbers and opsin expression [34] To reveal the underlying molecular mechanisms of this observation, we here used comparative transcriptome analysis of the developing whole eye, including (premature) retinal photoreceptors, in animals from the same mutant line and WT. Upon analyzing gene expression changes in WT eyes along development, we found that 'Visual perception' and 'Sensory perception of light stimulus' are the major GO terms enriched in the genes whose expression increases from E15 to P3 (Supplementary Figure S6, see also Figure S5a, c). This indicates that cone and rod genes provide a substantial contribution to the characterized transcriptome and thus that our analyses are relevant for identifying related changes in the mutants.

Several groups previously reported a relationship between clock gene mutation(s) and abnormal eye/retinal development $[28-31,34,35,65,66]$ but most frequently the underlying mechanisms have not been fully elucidated. Our data (Figure 1) suggests that regulatory mechanisms of retinal development and 
differentiation driven by the clock are already at play at E13. However, it is at P3 that the gene expression changes induced by the absence of PER1 and PER2 are maximal in our transcriptome study (Figure 3, Figure 7c). It has been described that de-repression of cell type-specific differentiation programs is the major epigenetic change occurring along with retinal development [67]. In this context of global genome derepression, and since clock genes are modulators of gene expression programs, it is not surprising that the effects of clock perturbation are more pronounced at later (P3) than earlier (E15, E18) stages. In addition, expression of Clock and Bmal1 peaks at P3 (Figure 1). Thus, absence of repression by PER1/PER2 on BMAL1/CLOCK target genes, might have its maximal effects at P3 as well. For instance, the upregulation of Per3 mRNA in mutant vs WT eyes is striking (FC $>2.5$ at P3 but also FC $>1.9$ at E15, and $>1.8$ at E18). This observation could possibly also be due to a compensatory developmental effect induced by Per1/Per2 mutation. Further studies are needed to shed light on these hypotheses.

Our results also raise a more general question whether the DEGs we identified are (in-)direct targets of the BMAL1/CLOCK complex. Therefore, we compared our dataset with a list of 2458 binding sites/1905 target genes for the BMAL1/CLOCK complex, previously identified in the liver by Trott and Menet [39]. A limitation of this study is that we cannot exclude that eye-specific targets of BMAL1/CLOCK are not represented in the list of liver targets. We found that $5.7 \%$ of all our DEGs overlap with this list. However, we did not observe an enrichment for BMAL1/CLOCK targets in our DEG set (one-sided Fisher's exact test, $p=0.74$ ). This indicates that the genes found differentially expressed between WT and mutant eyes contain both direct (for instance $D b p, N r 1 d 2$ ) and (essentially) indirect targets of the clock.

Adaptation of vision to the light/dark cycle involves the rhythmic expression of phototransduction components such as opsins $[11,12]$ and transducin [68], including their shuttling between the inner segment and the outer segment [68] and the post-translational modulation of ion channel sensitivity [69]. Mechanisms underlying rhythmic transcription of genes from the phototransduction cascade are not yet completely understood. In zebrafish, key developmental, retina-specific transcription factors driving photoreceptor differentiation, such as NR2E3, are co-regulated by the circadian clock in the adult for the control of daily photoreceptor physiology [70]. In our study, the upregulation of phototransduction related genes (19.7\% of the genes associated with this pathway) in Per1 ${ }^{-/}$Per2 ${ }^{B r d m} 1$ mice supports the idea that an important part of the phototransduction components might also be the (direct or indirect) target of the mammalian clock. 
It is presently unknown how the clock might control phototransduction gene expression in the mammalian retina. Our (Ingenuity pathway) analysis indicates that, in accordance with the literature [71, 72], NRL and $\mathrm{CRX}$ are the major regulators of the upregulated genes at P3. Nr2e3, which acts as a co-activator of rodgenes with NRL, CRX and REV-ERB $\alpha$ (NR1D1) [73], has a FC > 1.6 at P3 in our Per1 ${ }^{-/-}$Per2 ${ }^{\text {Brdm1 }}$ mutant eyes. Nr2e3 is a photoreceptor-specific nuclear receptor (PNR) that represses cone-specific genes and activates several rod-specific genes during mammalian retinal development [74]. When overexpressed in developing photoreceptors, $\mathrm{Nr} 2 \mathrm{e} 3$ drives their differentiation towards a rod fate at the expense of cones [75], whereas its absence may lead to "hyperfunction" of S-cones [76, 77]. Thus, Nr2e3 mutations cause enhanced Scone syndrome among humans [76]. In the mutant eyes at P3 we find an increase in expression of rhodopsin (FC>1.6) and strong decrease (2 fold at E18, 2.4-fold at P3) for s-opsin, in agreement with the previously observed phenotype [34]. In addition, expression of Nr2e3 was also shown to be rhythmic in the $24 \mathrm{~h}$ transcriptome of mouse eye globes [15] and in zebrafish adult retinas [70], suggesting it is regulated by the clock. Combining our data with those of the literature, we speculate that NR2E3-mediated regulation might be one of the causes of the morphological/functional changes in the phototransduction machinery observed in the mutant at P3.

Another transcription factor gene displaying upregulation $(F C>1.5)$ in the mutant eye at P3 is Rorc. The Rorc gene codes for the ROR gamma clock factor and is itself a target of Nr2e3 during retinal development $[31,78]$. It was also previously determined that loss of Rora, one of its paralogs, leads to defective cone differentiation and reduced expression of blue, green opsin as well as cone arrestin [79]. In the absence of Rorb, complete loss of rods and over-production of blue cones occurs during retinal development [80]. It will be interesting to understand if the retinal architecture is disrupted in the absence/mutation of Rorc as well. Obviously, further studies are needed to determine the precise mechanisms involved.

Effects of clock alteration on blue cones was also described previously [35]. More specifically, photoreceptor-specific loss of Bmal1 induces extended distribution of the s-opsin expressing cones along the dorso-ventral axis. This was shown to involve differential thyroid hormone signaling through an effect on Dio2 expression [35]. However, we did not detect any significant alteration of the expression of thyroid hormone pathway modulators in the Per1 -/- Per2 ${ }^{B r d m 1}$ mutants at any of the studied ages. It might be that such alterations occur later in development. Indeed, Dio2 expression is detectable after P5, similar to that of m-opsin [35]. In contrast, we found a decrease in the expression levels of s-opsin at E18 and P3, which is retained in the adult [34]. This is similar to the phenotype induced by a photoreceptor-specific knockout of Per2 [35]. Our data thus indicate that the clock effects on cone generation is two-fold: an early effect 
leading to reduced s-opsin expression/s-cone differentiation and a later, thyroid hormone dependent, effect on their dorso-ventral gradient.

In our study, the cell cycle appears perturbed in the eyes from Per1 /- Per2 ${ }^{\text {Brdm1 }}$ animals at P3 (Figure 7 and Supplementary Figure S5d), an age at which a substantial part of precursors for late-born retinal cell types are still dividing (reviewed in $[43,58])$. The link between circadian clock and cell cycle regulation has long been established [81]. However, there are only few studies of clock regulation, cell division and cellular differentiation together. The relationship between Bmal1 loss and cell cycle arrest, with an upregulation of $p 21$ and a block in the G1 phase, was previously studied in the hair follicles [82]. The gene expression levels of Wee1 (cell cycle checkpoint kinase) and p21 (cyclin-dependent kinase inhibitor) are also regulated by Bmal1 in the liver [83]. Cry2 promotes the circadian regulation of myogenic differentiation and regeneration in the mouse [84] whereas Rev-Erbo (Nr1d1) acts as an inhibitor of the same processes [85]. Also, Clock gene silencing induced spontaneous differentiation of mouse embryonic stem cells indicating an earlier exit from their pluripotent state [86]. In general, cell-cycle specific markers have been strongly connected with retina development [87]. E2f family transcription factors [88, 89] and cyclin D1 [90-93] are well known to regulate embryonic aspects of retinal development. In the Per1 ${ }^{-/}$Per2 ${ }^{\text {Brdm1 } 1}$ eyes it might be that reduced expression of genes promoting cell division triggers faster cell cycle exit, which is likely to disturb the ratio between late-born to early-born cells as shown previously regarding cyclin D1 [90, 91]: here the overproduction of rod and cone markers at the expense of blue cones. Interestingly, conditional knockout of Bmal1 in the retina was recently shown to result in delayed cell-cycle exit and subsequent altered neurogenesis [28]. Therefore, we surmise to ensure proper generation of retinal cell types, the timing needs to be optimum and the circadian clock might be responsible for appropriate temporal expression patterns of cell cycle-related genes.

Finally, we still do not know exactly how the absence of Per1 and Per2 shuts down the expression of cell cycle-related genes discussed extensively above. Pathway analysis of transcriptome changes along development in WT eyes indicates a downregulation of genes belonging to the Wnt and Hippo signaling pathways between E15 and E18 (Supplementary Fig S5b). By contrast, these pathways are absent or underrepresented in the genes turned down between E15 and E18 in mutant eyes. Also, the PI3K-Akt signaling pathway, which includes many growth factors and associated receptors, is significant in mutants but absent in the WT (Supplementary Fig S5b). We hypothesize that the specific combination of signals turned off between E15 and E18 in the mutant eyes induces more massive depletion of cell cycle components and hence an earlier decrease in progenitor proliferation. Future studies are needed to make 
sure that the downregulation of these cell-cycle-related pathways occurs in the retina exclusively and not elsewhere in the eye.

\section{Conclusions}

This study provides new information about the role of the circadian clock in eye development. It more specifically points to signaling pathways potentially linking the clock, photoreceptor generation and control of the cell cycle in the developing retina. Moreover, our findings reveal potential mechanisms for the putative ocular effects of clock malfunction reinforcing the ubiquitous relevance of circadian clock in eye morphogenesis. More studies will be needed to determine the connection between the phenotype and retinal abnormalities.

\section{Author contributions}

Conceptualization: Udita Bagchi, Marie-Paule Felder-Schmittbuhl, Arthur A. Bergen

Software: Aldo Jongejan, Perry D. Moerland

Validation: Udita Bagchi, Cristina Sandu, Marie-Paule Felder-Schmittbuhl

Formal analysis: Udita Bagchi, Aldo Jongejan, Perry D. Moerland

Investigation: Udita Bagchi, Shumet Gegnaw

Resources: Udita Bagchi, Cristina Sandu, Nemanja Milićević, Jacoline B. ten Brink

Data curation: Udita Bagchi, Shumet Gegnaw, Cristina Sandu, Aldo Jongejan

Writing - original draft: Udita Bagchi

Writing - review \& editing: Udita Bagchi, Nemanja Milicevic, Perry D. Moerland, David Hicks, Marie-

Paule Felder-Schmittbuhl, Arthur A. Bergen

Visualization: Udita Bagchi, Cristina Sandu, Aldo Jongejan

Supervision: Marie-Paule Felder-Schmittbuhl, Perry D. Moerland, Arthur A. Bergen

Project administration: Udita Bagchi, Cristina Sandu, Marie-Paule Felder-Schmittbuhl

Funding acquisition: Marie-Paule Felder-Schmittbuhl, Arthur A. Bergen

\section{Acknowledgements}

The authors would like to thank $\operatorname{Dr}$ U. Albrecht (University of Fribourg, Switzerland) for providing the Per1 ${ }^{-}$Per2 ${ }^{\text {Brdm1 }}$ mice. This work was carried out in Academic Medical Center (AMC) Amsterdam, The Netherlands, in close cooperation with CNRS/University of Strasbourg, France. The authors confirm no conflict of interests in the execution of this work. 


\section{Funding}

This study was part of Neurotime Erasmus Mundus Project number: 520124-1-2011-1-FR-ERA MundusEPJD, FPA: 2012-0026, supported by a generous grant from the European Union. The funders had no role in study design, data collection and analysis, decision to publish or preparation of the manuscript.

\section{References}

[1] J.A. Mohawk, C.B. Green, J.S. Takahashi, Central and peripheral circadian clocks in mammals, Annu Rev Neurosci, 35 (2012) 445-462.

[2] J.S. Takahashi, Transcriptional architecture of the mammalian circadian clock, Nat Rev Genet, 18 (2017) 164-179.

[3] S.M. Reppert, D.R. Weaver, Coordination of circadian timing in mammals, Nature, 418 (2002) 935941.

[4] S.H. Yoo, S. Yamazaki, P.L. Lowrey, K. Shimomura, C.H. Ko, E.D. Buhr, S.M. Siepka, H.K. Hong, W.J. Oh, O.J. Yoo, M. Menaker, J.S. Takahashi, PERIOD2::LUCIFERASE real-time reporting of circadian dynamics reveals persistent circadian oscillations in mouse peripheral tissues, Proc Natl Acad Sci U S A, 101 (2004) 5339-5346.

[5] G. Tosini, M. Menaker, Circadian rhythms in cultured mammalian retina, Science, 272 (1996) 419-421.

[6] M.P. Felder-Schmittbuhl, E.D. Buhr, O. Dkhissi-Benyahya, D. Hicks, S.N. Peirson, C.P. Ribelayga, C. Sandu, R. Spessert, G. Tosini, Ocular Clocks: Adapting Mechanisms for Eye Functions and Health, Invest Ophthalmol Vis Sci, 59 (2018) 4856-4870.

[7] H.S. Lee, J.L. Nelms, M. Nguyen, R. Silver, M.N. Lehman, The eye is necessary for a circadian rhythm in the suprachiasmatic nucleus, Nat Neurosci, 6 (2003) 111-112.

[8] S. Yamazaki, V. Alones, M. Menaker, Interaction of the retina with suprachiasmatic pacemakers in the control of circadian behavior, J Biol Rhythms, 17 (2002) 315-329.

[9] D.G. McMahon, P.M. luvone, G. Tosini, Circadian organization of the mammalian retina: from gene regulation to physiology and diseases, Prog Retin Eye Res, 39 (2014) 58-76.

[10] H.C. Marie-Paule Felder-Schmittbuhl, Ouria Dkhissi-Benyahya, The retinal clock in mammals: role in health and disease, ChronoPhysiology and Therapy, (2017) 12.

[11] C. Bobu, C. Sandu, V. Laurent, M.P. Felder-Schmittbuhl, D. Hicks, Prolonged light exposure induces widespread phase shifting in the circadian clock and visual pigment gene expression of the Arvicanthis ansorgei retina, Mol Vis, 19 (2013) 1060-1073.

[12] M. von Schantz, R.J. Lucas, R.G. Foster, Circadian oscillation of photopigment transcript levels in the mouse retina, Brain Res Mol Brain Res, 72 (1999) 108-114.

[13] A.R. Barnard, S. Hattar, M.W. Hankins, R.J. Lucas, Melanopsin regulates visual processing in the mouse retina, Curr Biol, 16 (2006) 389-395.

[14] M.A. Cameron, A.R. Barnard, R.J. Lucas, The electroretinogram as a method for studying circadian rhythms in the mammalian retina, J Genet, 87 (2008) 459-466.

[15] K.F. Storch, C. Paz, J. Signorovitch, E. Raviola, B. Pawlyk, T. Li, C.J. Weitz, Intrinsic circadian clock of the mammalian retina: importance for retinal processing of visual information, Cell, 130 (2007) 730-741.

[16] C. Bobu, D. Hicks, Regulation of retinal photoreceptor phagocytosis in a diurnal mammal by circadian clocks and ambient lighting, Invest Ophthalmol Vis Sci, 50 (2009) 3495-3502.

[17] M.M. LaVail, Circadian nature of rod outer segment disc shedding in the rat, Invest Ophthalmol Vis Sci, 19 (1980) 407-411. 
[18] D.T. Organisciak, R.M. Darrow, L. Barsalou, R.K. Kutty, B. Wiggert, Circadian-dependent retinal light damage in rats, Invest Ophthalmol Vis Sci, 41 (2000) 3694-3701.

[19] Y. Kikkawa, Diurnal variation in corneal thickness, Exp Eye Res, 15 (1973) 1-9.

[20] Y. Xue, P. Liu, H. Wang, C. Xiao, C. Lin, J. Liu, D. Dong, T. Fu, Y. Yang, Z. Wang, H. Pan, J. Chen, Y. Li, D. Cai, Z. Li, Modulation of Circadian Rhythms Affects Corneal Epithelium Renewal and Repair in Mice, Invest Ophthalmol Vis Sci, 58 (2017) 1865-1874.

[21] S. Tsuchiya, E.D. Buhr, T. Higashide, K. Sugiyama, R.N. Van Gelder, Light entrainment of the murine intraocular pressure circadian rhythm utilizes non-local mechanisms, PLoS One, 12 (2017) e0184790.

[22] D.C. Lozano, A.T. Hartwick, M.D. Twa, Circadian rhythm of intraocular pressure in the adult rat, Chronobiol Int, 32 (2015) 513-523.

[23] Y. Kobayashi, Z. Ye, T.K. Hensch, Clock genes control cortical critical period timing, Neuron, 86 (2015) 264-275.

[24] M. Mirmiran, L. Bernardo, S.L. Jenkins, X.H. Ma, J.T. Brenna, P.W. Nathanielsz, Growth, neurobehavioral and circadian rhythm development in newborn baboons, Pediatr Res, 49 (2001) 673677.

[25] A. Sumova, M. Sladek, L. Polidarova, M. Novakova, P. Houdek, Circadian system from conception till adulthood, Prog Brain Res, 199 (2012) 83-103.

[26] S. Honma, Development of the mammalian circadian clock, Eur J Neurosci, (2018).

[27] X. Liu, Z. Zhang, C.P. Ribelayga, Heterogeneous expression of the core circadian clock proteins among neuronal cell types in mouse retina, PLoS One, 7 (2012) e50602.

[28] O.B. Sawant, V.K. Jidigam, R.D. Fuller, O.F. Zucaro, C. Kpegba, M. Yu, N.S. Peachey, S. Rao, The circadian clock gene Bmal1 is required to control the timing of retinal neurogenesis and lamination of Muller glia in the mouse retina, FASEB J, (2019) fj201801832RR.

[29] K. Baba, I. Piano, P. Lyuboslavsky, M.A. Chrenek, J.T. Sellers, S. Zhang, C. Gargini, L. He, G. Tosini, P.M. luvone, Removal of clock gene Bmal1 from the retina affects retinal development and accelerates cone photoreceptor degeneration during aging, Proc Natl Acad Sci U S A, 115 (2018) 13099-13104. [30] O. Ait-Hmyed Hakkari, N. Acar, E. Savier, P. Spinnhirny, M. Bennis, M.P. Felder-Schmittbuhl, J. Mendoza, D. Hicks, Rev-Erbalpha modulates retinal visual processing and behavioral responses to light, FASEB J, 30 (2016) 3690-3701.

[31] N.J. Mollema, Y. Yuan, A.S. Jelcick, A.J. Sachs, D. von Alpen, D. Schorderet, P. Escher, N.B. Haider, Nuclear receptor Rev-erb alpha (Nr1d1) functions in concert with Nr2e3 to regulate transcriptional networks in the retina, PLoS One, 6 (2011) e17494.

[32] B. Zheng, U. Albrecht, K. Kaasik, M. Sage, W. Lu, S. Vaishnav, Q. Li, Z.S. Sun, G. Eichele, A. Bradley, C.C. Lee, Nonredundant roles of the mPer1 and mPer2 genes in the mammalian circadian clock, Cell, 105 (2001) 683-694.

[33] B. Zheng, D.W. Larkin, U. Albrecht, Z.S. Sun, M. Sage, G. Eichele, C.C. Lee, A. Bradley, The mPer2 gene encodes a functional component of the mammalian circadian clock, Nature, 400 (1999) 169-173. [34] O. Ait-Hmyed, M.P. Felder-Schmittbuhl, M. Garcia-Garrido, S. Beck, C. Seide, V. Sothilingam, N. Tanimoto, M. Seeliger, M. Bennis, D. Hicks, Mice lacking Period 1 and Period 2 circadian clock genes exhibit blue cone photoreceptor defects, Eur J Neurosci, 37 (2013) 1048-1060.

[35] O.B. Sawant, A.M. Horton, O.F. Zucaro, R. Chan, V.L. Bonilha, I.S. Samuels, S. Rao, The Circadian Clock Gene Bmal1 Controls Thyroid Hormone-Mediated Spectral Identity and Cone Photoreceptor Function, Cell Rep, 21 (2017) 692-706.

[36] J.C.Y. Wong, N.J. Smyllie, G.T. Banks, C.A. Pothecary, A.R. Barnard, E.S. Maywood, A. Jagannath, S. Hughes, G.T.J. van der Horst, R.E. MacLaren, M.W. Hankins, M.H. Hastings, P.M. Nolan, R.G. Foster, S.N. Peirson, Differential roles for cryptochromes in the mammalian retinal clock, FASEB J, 32 (2018) 43024314. 
[37] A. Chaix, A. Zarrinpar, S. Panda, The circadian coordination of cell biology, J Cell Biol, 215 (2016) 1525.

[38] S. Panda, M.P. Antoch, B.H. Miller, A.I. Su, A.B. Schook, M. Straume, P.G. Schultz, S.A. Kay, J.S. Takahashi, J.B. Hogenesch, Coordinated transcription of key pathways in the mouse by the circadian clock, Cell, 109 (2002) 307-320.

[39] A.J. Trott, J.S. Menet, Regulation of circadian clock transcriptional output by CLOCK:BMAL1, PLoS Genet, 14 (2018) e1007156.

[40] J.R. Beytebiere, B.J. Greenwell, A. Sahasrabudhe, J.S. Menet, Clock-controlled rhythmic transcription: is the clock enough and how does it work?, Transcription, 10 (2019) 212-221.

[41] R. Zhang, N.F. Lahens, H.I. Ballance, M.E. Hughes, J.B. Hogenesch, A circadian gene expression atlas in mammals: implications for biology and medicine, Proc Natl Acad Sci U S A, 111 (2014) 16219-16224. [42] L.S. Mure, H.D. Le, G. Benegiamo, M.W. Chang, L. Rios, N. Jillani, M. Ngotho, T. Kariuki, O. DkhissiBenyahya, H.M. Cooper, S. Panda, Diurnal transcriptome atlas of a primate across major neural and peripheral tissues, Science, 359 (2018).

[43] C.L. Cepko, C.P. Austin, X. Yang, M. Alexiades, D. Ezzeddine, Cell fate determination in the vertebrate retina, Proc Natl Acad Sci U S A, 93 (1996) 589-595.

[44] L.D. Carter-Dawson, M.M. LaVail, Rods and cones in the mouse retina. II. Autoradiographic analysis of cell generation using tritiated thymidine, J Comp Neurol, 188 (1979) 263-272.

[45] C. Sandu, D. Hicks, M.P. Felder-Schmittbuhl, Rat photoreceptor circadian oscillator strongly relies on lighting conditions, Eur J Neurosci, 34 (2011) 507-516.

[46] J. Hellemans, G. Mortier, A. De Paepe, F. Speleman, J. Vandesompele, qBase relative quantification framework and software for management and automated analysis of real-time quantitative PCR data, Genome Biol, 8 (2007) R19.

[47] H. Adachi, H. Tominaga, Y. Maruyama, K. Yoneda, K. Maruyama, K. Yoshii, S. Kinoshita, M. Nakano, K. Tashiro, Stage-specific reference genes significant for quantitative PCR during mouse retinal development, Genes Cells, 20 (2015) 625-635.

[48] S. Sayols, D. Scherzinger, H. Klein, dupRadar: a Bioconductor package for the assessment of PCR artifacts in RNA-Seq data, BMC Bioinformatics, 17 (2016) 428.

[49] A.M. Bolger, M. Lohse, B. Usadel, Trimmomatic: a flexible trimmer for Illumina sequence data, Bioinformatics, 30 (2014) 2114-2120.

[50] D. Kim, B. Langmead, S.L. Salzberg, HISAT: a fast spliced aligner with low memory requirements, Nat Methods, 12 (2015) 357-360.

[51] S. Anders, P.T. Pyl, W. Huber, HTSeq--a Python framework to work with high-throughput sequencing data, Bioinformatics, 31 (2015) 166-169.

[52] M.D. Robinson, D.J. McCarthy, G.K. Smyth, edgeR: a Bioconductor package for differential expression analysis of digital gene expression data, Bioinformatics, 26 (2010) 139-140.

[53] M.E. Ritchie, B. Phipson, D. Wu, Y. Hu, C.W. Law, W. Shi, G.K. Smyth, limma powers differential expression analyses for RNA-sequencing and microarray studies, Nucleic Acids Res, 43 (2015) e47. [54] M.D. Robinson, A. Oshlack, A scaling normalization method for differential expression analysis of RNA-seq data, Genome Biol, 11 (2010) R25.

[55] C.W. Law, Y. Chen, W. Shi, G.K. Smyth, voom: Precision weights unlock linear model analysis tools for RNA-seq read counts, Genome Biol, 15 (2014) R29.

[56] S.M.-I. Consortium, A comprehensive assessment of RNA-seq accuracy, reproducibility and information content by the Sequencing Quality Control Consortium, Nat Biotechnol, 32 (2014) 903-914. [57] A. Bennis, J.B. Ten Brink, P.D. Moerland, V.M. Heine, A.A. Bergen, Comparative gene expression study and pathway analysis of the human iris- and the retinal pigment epithelium, PLoS One, 12 (2017) e0182983. 
[58] E.M. Levine, E.S. Green, Cell-intrinsic regulators of proliferation in vertebrate retinal progenitors, Semin Cell Dev Biol, 15 (2004) 63-74.

[59] R.W. Logan, C.A. McClung, Rhythms of life: circadian disruption and brain disorders across the lifespan, Nat Rev Neurosci, 20 (2019) 49-65.

[60] J. Zhao, X. Zhou, Q. Tang, R. Yu, S. Yu, Y. Long, C. Cao, J. Han, A. Shi, J.J. Mao, X. Chen, L. Chen, BMAL1 Deficiency Contributes to Mandibular Dysplasia by Upregulating MMP3, Stem Cell Reports, 10 (2018) 180-195.

[61] X. Zhou, R. Yu, Y. Long, J. Zhao, S. Yu, Q. Tang, L. Chen, BMAL1 deficiency promotes skeletal mandibular hypoplasia via OPG downregulation, Cell Prolif, 51 (2018) e12470.

[62] L.D. Jensen, Z. Cao, M. Nakamura, Y. Yang, L. Brautigam, P. Andersson, Y. Zhang, E. Wahlberg, T. Lanne, K. Hosaka, Y. Cao, Opposing effects of circadian clock genes bmal1 and period 2 in regulation of VEGF-dependent angiogenesis in developing zebrafish, Cell Rep, 2 (2012) 231-241.

[63] C.M. McQueen, E.E. Schmitt, T.R. Sarkar, J. Elswood, R.P. Metz, D. Earnest, M. Rijnkels, W.W. Porter, PER2 regulation of mammary gland development, Development, 145 (2018).

[64] Z. Li, L. Ruan, S. Lin, G.K. Gittes, Clock controls timing of mouse pancreatic differentiation through regulation of Wnt- and Notch-based and cell division components, Biochem Biophys Res Commun, 359 (2007) 491-496.

[65] R.A. Stone, A.M. McGlinn, R. Chakraborty, D.C. Lee, V. Yang, A. Elmasri, E. Landis, J. Shaffer, P.M. luvone, X. Zheng, A. Sehgal, M.T. Pardue, Altered ocular parameters from circadian clock gene disruptions, PLoS One, 14 (2019) e0217111.

[66] R.A. Stone, M.T. Pardue, P.M. luvone, T.S. Khurana, Pharmacology of myopia and potential role for intrinsic retinal circadian rhythms, Exp Eye Res, 114 (2013) 35-47.

[67] I. Aldiri, B. Xu, L. Wang, X. Chen, D. Hiler, L. Griffiths, M. Valentine, A. Shirinifard, S. Thiagarajan, A. Sablauer, M.E. Barabas, J. Zhang, D. Johnson, S. Frase, X. Zhou, J. Easton, J. Zhang, E.R. Mardis, R.K. Wilson, J.R. Downing, M.A. Dyer, P. St. Jude Children's Research Hospital-Washington University Pediatric Cancer Genome, The Dynamic Epigenetic Landscape of the Retina During Development, Reprogramming, and Tumorigenesis, Neuron, 94 (2017) 550-568 e510.

[68] J.N. Pearring, R.Y. Salinas, S.A. Baker, V.Y. Arshavsky, Protein sorting, targeting and trafficking in photoreceptor cells, Prog Retin Eye Res, 36 (2013) 24-51.

[69] G.Y. Ko, M.L. Ko, S.E. Dryer, Circadian regulation of cGMP-gated channels of vertebrate cone photoreceptors: role of cAMP and Ras, J Neurosci, 24 (2004) 1296-1304.

[70] R. Laranjeiro, D. Whitmore, Transcription factors involved in retinogenesis are co-opted by the circadian clock following photoreceptor differentiation, Development, 141 (2014) 2644-2656.

[71] J.C. Corbo, K.A. Lawrence, M. Karlstetter, C.A. Myers, M. Abdelaziz, W. Dirkes, K. Weigelt, M. Seifert, V. Benes, L.G. Fritsche, B.H. Weber, T. Langmann, CRX ChIP-seq reveals the cis-regulatory architecture of mouse photoreceptors, Genome Res, 20 (2010) 1512-1525.

[72] H. Hao, D.S. Kim, B. Klocke, K.R. Johnson, K. Cui, N. Gotoh, C. Zang, J. Gregorski, L. Gieser, W. Peng, Y. Fann, M. Seifert, K. Zhao, A. Swaroop, Transcriptional regulation of rod photoreceptor homeostasis revealed by in vivo NRL targetome analysis, PLoS Genet, 8 (2012) e1002649.

[73] H. Cheng, H. Khanna, E.C. Oh, D. Hicks, K.P. Mitton, A. Swaroop, Photoreceptor-specific nuclear receptor NR2E3 functions as a transcriptional activator in rod photoreceptors, Hum Mol Genet, 13 (2004) $1563-1575$.

[74] G.H. Peng, O. Ahmad, F. Ahmad, J. Liu, S. Chen, The photoreceptor-specific nuclear receptor Nr2e3 interacts with $\mathrm{Crx}$ and exerts opposing effects on the transcription of rod versus cone genes, Hum Mol Genet, 14 (2005) 747-764.

[75] H. Cheng, T.S. Aleman, A.V. Cideciyan, R. Khanna, S.G. Jacobson, A. Swaroop, In vivo function of the orphan nuclear receptor NR2E3 in establishing photoreceptor identity during mammalian retinal development, Hum Mol Genet, 15 (2006) 2588-2602. 
[76] N.B. Haider, S.G. Jacobson, A.V. Cideciyan, R. Swiderski, L.M. Streb, C. Searby, G. Beck, R. Hockey, D.B. Hanna, S. Gorman, D. Duhl, R. Carmi, J. Bennett, R.G. Weleber, G.A. Fishman, A.F. Wright, E.M. Stone, V.C. Sheffield, Mutation of a nuclear receptor gene, NR2E3, causes enhanced S cone syndrome, a disorder of retinal cell fate, Nat Genet, 24 (2000) 127-131.

[77] N.B. Haider, J.K. Naggert, P.M. Nishina, Excess cone cell proliferation due to lack of a functional NR2E3 causes retinal dysplasia and degeneration in rd7/rd7 mice, Hum Mol Genet, 10 (2001) 1619-1626. [78] N.B. Haider, N. Mollema, M. Gaule, Y. Yuan, A.J. Sachs, A.M. Nystuen, J.K. Naggert, P.M. Nishina, Nr2e3-directed transcriptional regulation of genes involved in photoreceptor development and cell-type specific phototransduction, Exp Eye Res, 89 (2009) 365-372.

[79] H. Fujieda, R. Bremner, A.J. Mears, H. Sasaki, Retinoic acid receptor-related orphan receptor alpha regulates a subset of cone genes during mouse retinal development, J Neurochem, 108 (2009) 91-101. [80] L. Jia, E.C. Oh, L. Ng, M. Srinivas, M. Brooks, A. Swaroop, D. Forrest, Retinoid-related orphan nuclear receptor RORbeta is an early-acting factor in rod photoreceptor development, Proc Natl Acad Sci U S A, 106 (2009) 17534-17539.

[81] S. Masri, M. Cervantes, P. Sassone-Corsi, The circadian clock and cell cycle: interconnected biological circuits, Curr Opin Cell Biol, 25 (2013) 730-734.

[82] K.K. Lin, V. Kumar, M. Geyfman, D. Chudova, A.T. Ihler, P. Smyth, R. Paus, J.S. Takahashi, B. Andersen, Circadian clock genes contribute to the regulation of hair follicle cycling, PLoS Genet, 5 (2009) e1000573. [83] T. Matsuo, S. Yamaguchi, S. Mitsui, A. Emi, F. Shimoda, H. Okamura, Control mechanism of the circadian clock for timing of cell division in vivo, Science, 302 (2003) 255-259.

[84] M. Lowe, J. Lage, E. Paatela, D. Munson, R. Hostager, C. Yuan, N. Katoku-Kikyo, M. Ruiz-Estevez, Y. Asakura, J. Staats, M. Qahar, M. Lohman, A. Asakura, N. Kikyo, Cry2 Is Critical for Circadian Regulation of Myogenic Differentiation by Bclaf1-Mediated mRNA Stabilization of Cyclin D1 and Tmem176b, Cell Rep, 22 (2018) 2118-2132.

[85] S. Chatterjee, H. Yin, W. Li, J. Lee, V.K. Yechoor, K. Ma, The Nuclear Receptor and Clock Repressor Rev-erbalpha Suppresses Myogenesis, Sci Rep, 9 (2019) 4585.

[86] C. Lu, Y. Yang, R. Zhao, B. Hua, C. Xu, Z. Yan, N. Sun, R. Qian, Role of circadian gene Clock during differentiation of mouse pluripotent stem cells, Protein Cell, 7 (2016) 820-832.

[87] M.A. Dyer, C.L. Cepko, Regulating proliferation during retinal development, Nat Rev Neurosci, 2 (2001) 333-342.

[88] L. Dagnino, C.J. Fry, S.M. Bartley, P. Farnham, B.L. Gallie, R.A. Phillips, Expression patterns of the E2F family of transcription factors during mouse nervous system development, Mech Dev, 66 (1997) 13-25. [89] L. Vuong, D.E. Brobst, A. Saadi, I. Ivanovic, M.R. Al-Ubaidi, Pattern of expression of p53, its family members, and regulators during early ocular development and in the post-mitotic retina, Invest Ophthalmol Vis Sci, 53 (2012) 4821-4831.

[90] G. Das, Y. Choi, P. Sicinski, E.M. Levine, Cyclin D1 fine-tunes the neurogenic output of embryonic retinal progenitor cells, Neural Dev, 4 (2009) 15.

[91] G. Das, A.M. Clark, E.M. Levine, Cyclin D1 inactivation extends proliferation and alters histogenesis in the postnatal mouse retina, Dev Dyn, 241 (2012) 941-952.

[92] V. Fantl, G. Stamp, A. Andrews, I. Rosewell, C. Dickson, Mice lacking cyclin D1 are small and show defects in eye and mammary gland development, Genes Dev, 9 (1995) 2364-2372.

[93] C. Ma, D. Papermaster, C.L. Cepko, A unique pattern of photoreceptor degeneration in cyclin D1 mutant mice, Proc Natl Acad Sci U S A, 95 (1998) 9938-9943. 


\section{Figure legends}

Fig 1 Relative mRNA expression profiles of circadian clock genes in the WT whole eyes across eye developmental time points ( $n=3-4 / a g e)$. Bar graphs show mean \pm SEM mRNA expression levels of (a) Arntl (Bmal1) (b) Clock (c) Per1 (d) Per2 (e) Per3 (f) Cry1 (g) Cry2 (h) Nr1d1 and (i) Rorb in the whole eyes relative to E13. Arntl (Bmal1) and Rorb mRNA reached a peak just after birth around P3, Clock gene expression increases steadily from E13 until P3, Per1, Cry1 and Per2 expression peaked at birth (P0), Per3 and Cry2 expression peaks only around P13 and P24. Nr1d1 has an increasing expression pattern between E13 and P24. One-Way ANOVA was performed and statistical significance is indicated by $p$-values. Values from individual samples are shown as dots. Results from post-hoc analysis are presented in the Supplementary Table S2.

Fig 2 Principal component analysis of eye transcriptomes in WT and mutant eyes at E15, E18 and P3. The percentage with which a principal component (PC) accounts for the variability in the data is indicated on the corresponding axis. Each circle represents an individual RNA sample from WT or mutants under LD conditions at ZT4 for three developmental time points (E15, E18 and P3) ( $n=4 /$ age/genotype).

Fig 3 (a) Venn diagram of differentially expressed genes in mutant versus WT eyes. The total numbers of differentially expressed genes (increased or decreased) in each age group and the overlaps are indicated. Upregulated (higher in mutant) genes are marked in red and downregulated (higher in WT) genes are marked in blue (adjusted p-value $<0.05$ and fold change $\leq-1.5$ or $\geq 1.5$ ). (b) Heatmap of clock and clockrelated gene expression during embryonic (E15 and E18) and postnatal (P3) ages of Per1 - Per2 $^{\text {Brdm1 }}$ mutants versus WT. Genes were clustered using Euclidean distance and complete linkage. Color-coding corresponds to z-score of logCPM values from blue (lowest expression) to red (highest expression). Per3, Nr1d2, Ciart, Bhlhe41, Rorc, Tef, Npas2 and Dbp displayed statistically significant changes between WT and mutants in at least one age group "*”. $\log C P M, \log _{2}$ counts per million.

Fig 4 Volcano plots for the genes found enriched in Per1 - Per2 ${ }^{\text {Brdm1 }}$ versus WT whole eyes at E15, E18 and P3. The plot shows the enrichment (x axis, log2-transformed fold change) of the genes in mutant versus WT eyes against the significance ( $y$ axis, $p$-value, - $\log 10$ scale). Genes with adjusted $p$-value $<0.05$ and fold change $\leq-1.5$ or $\geq 1.5$ are highlighted in grey. Selected genes are highlighted in red (upregulated) and blue (downregulated) for (a) E15 (complete list), (b) E18 and (c) P3 (eye and neuronal development related). Each circle represents an individual gene. 
Fig 5 (a) (b) and (c) Top-ranked canonical pathways at E15, E18 and P3 for DEG in Per1 /- Per2 ${ }^{\text {Brdm1 }}$ mutants versus WT. Bars represent the $-\log _{10}$ adjusted $p$-value (left $y$-axis). Orange line represents the ratio of the number of differentially expressed genes present in the canonical pathway to the total number of genes in the pathway (right $y$-axis). The green line indicates the threshold at an adjusted $p<0.01$.

Fig 6 Ingenuity pathway analysis identified Phototransduction Pathway to be associated with differentially expressed genes at P3. The phototransduction pathways of rods and cones are shown respectively in $\mathbf{a}$ and $\mathbf{b}$. Shapes of the network elements refer to the functional category of the gene product (indicated in the legend inset), pink indicates upregulation and green indicates downregulation (IPA All rights reserved).

Fig 7 IPA analysis of DEG at P3 showed enrichment in the (a) Cell Cycle Control of Chromosomal Replication genes and (b) Cyclins and Cell Cycle Regulation genes pathways. Significance for the differential expression between mutant and WT eyes is shown as $-\log _{10}$ (Adj p-value) (x-axis). There was an overall decrease in the expression of genes of pathways (a) and (b) in the mutant eyes at P3, except $C d k 18$ and Ppp2r2b. Up- and down-regulated genes are shown in red and blue respectively. (c) Heatmap of the differentially expressed genes of the cell-cycle related pathways during embryonic (E15 and E18) and postnatal (P3) ages of Per1 / Per2 ${ }^{\text {Brdm1 }}$ mutants versus WT. Genes were clustered using Euclidean distance and complete linkage. Color-coding corresponds to z-score of logCPM values from blue (lowest expression) to red (highest expression). E2f8, Ccnd1, Ccnb1, E2f2, Fanca, Kif11, Bard1, Espl1, Mcm2, Pole, Plk1, E2f7, Cdt1, Brip1, Cdc6, Mcm3, Cdc45, Mcm6, Top2a displayed pronounced downregulation in the mutants at P3 while Ppp2r2b, Cdk18, Plk5 had higher gene expression levels. Mlh1 stands out with an unusual, increased, expression pattern throughout development in the mutants. 

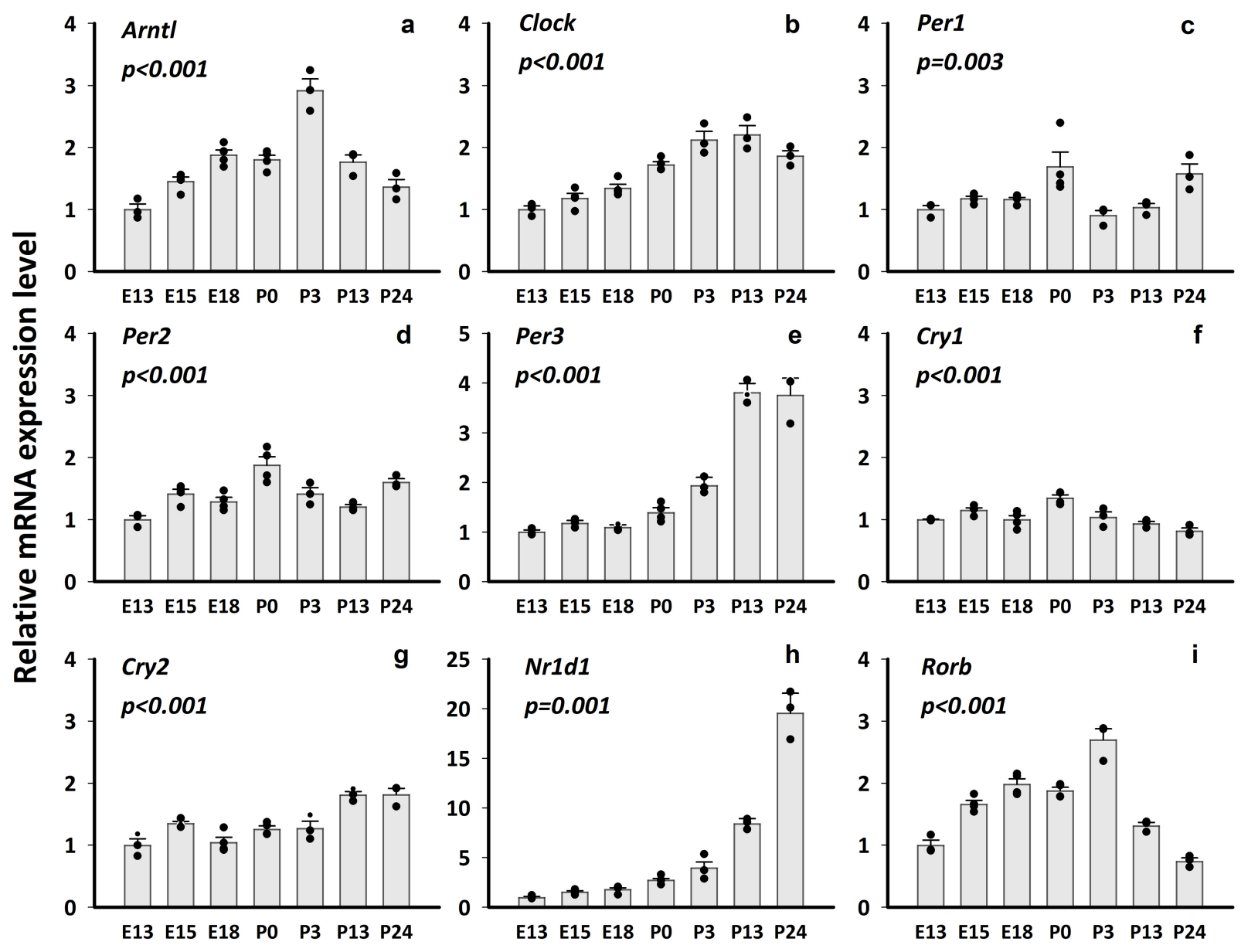

Age (days) 


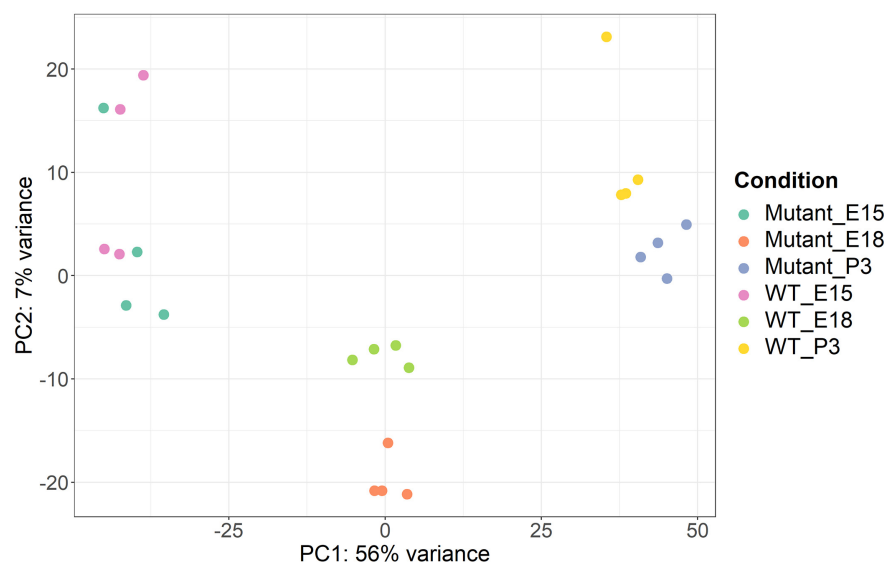


a

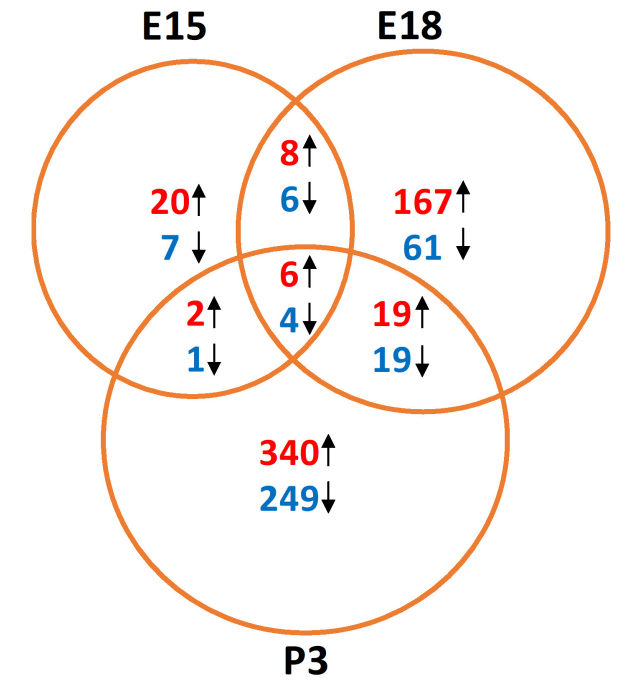

b

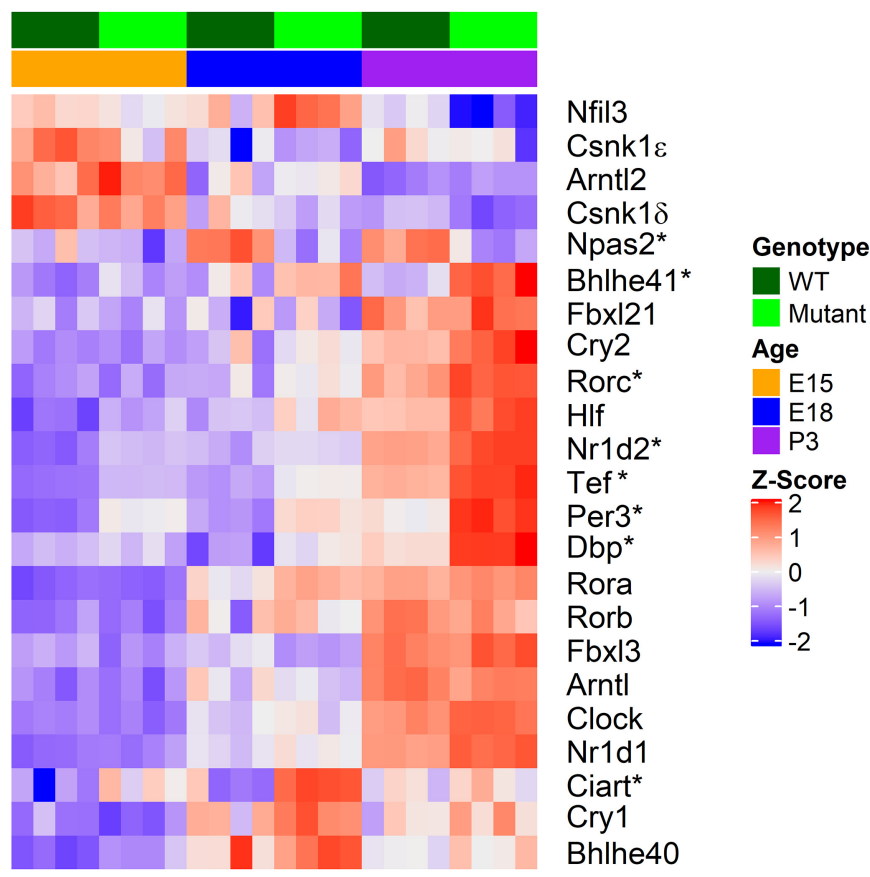



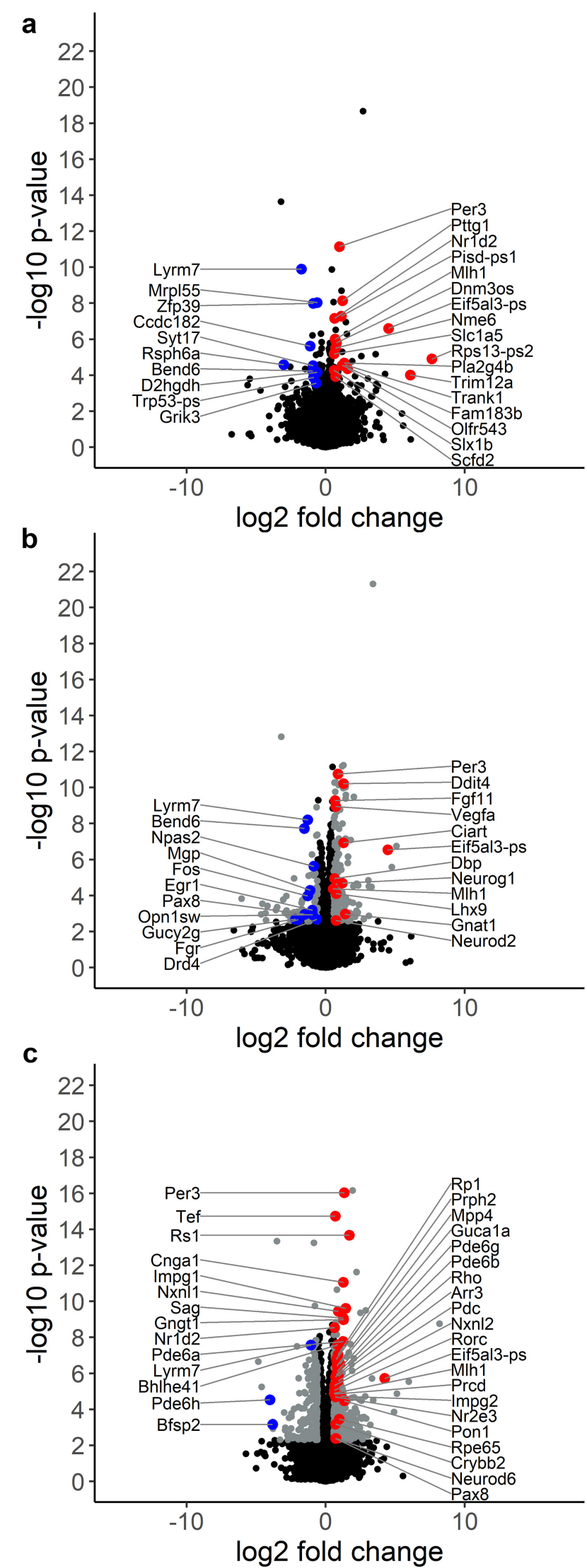

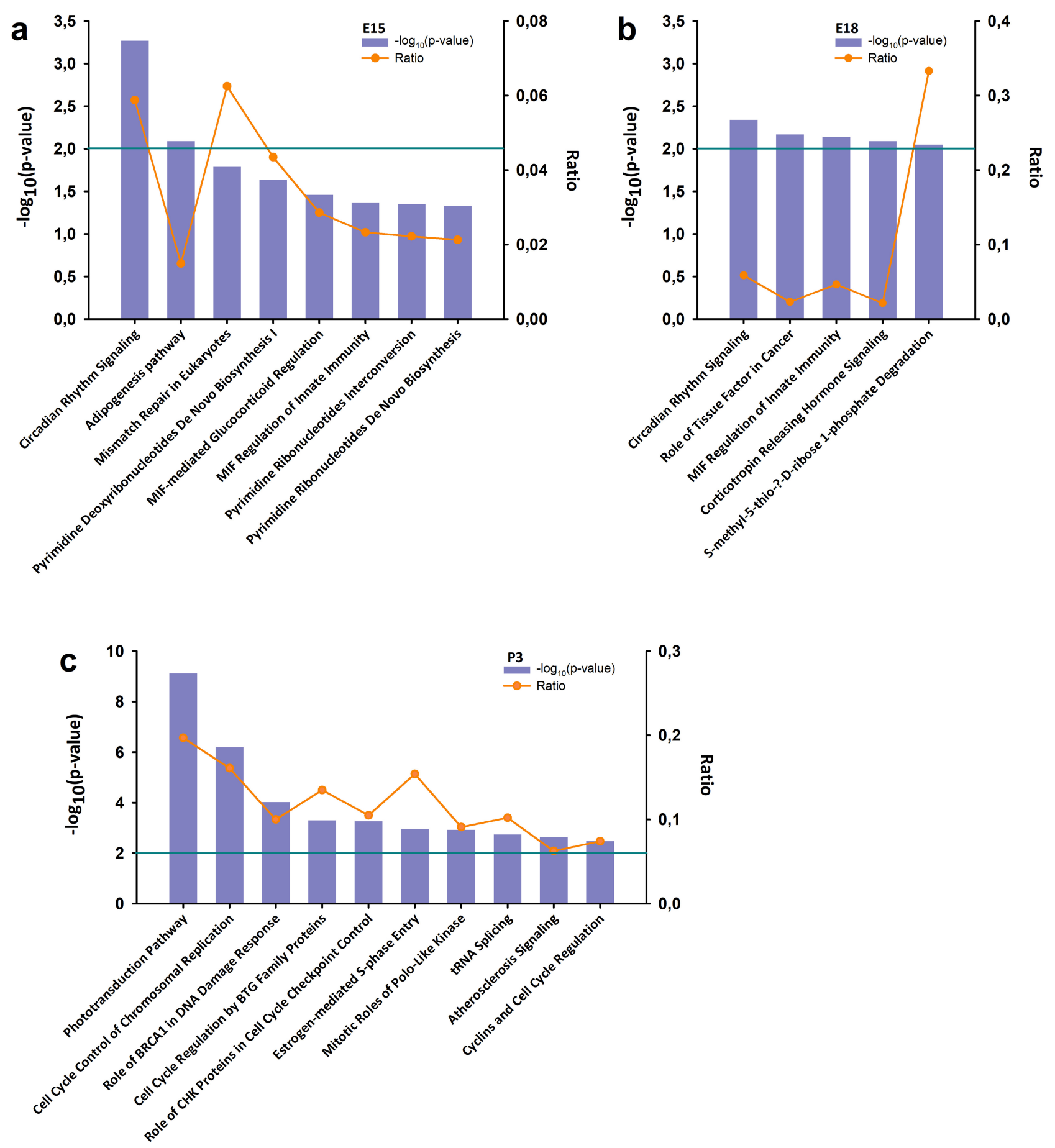

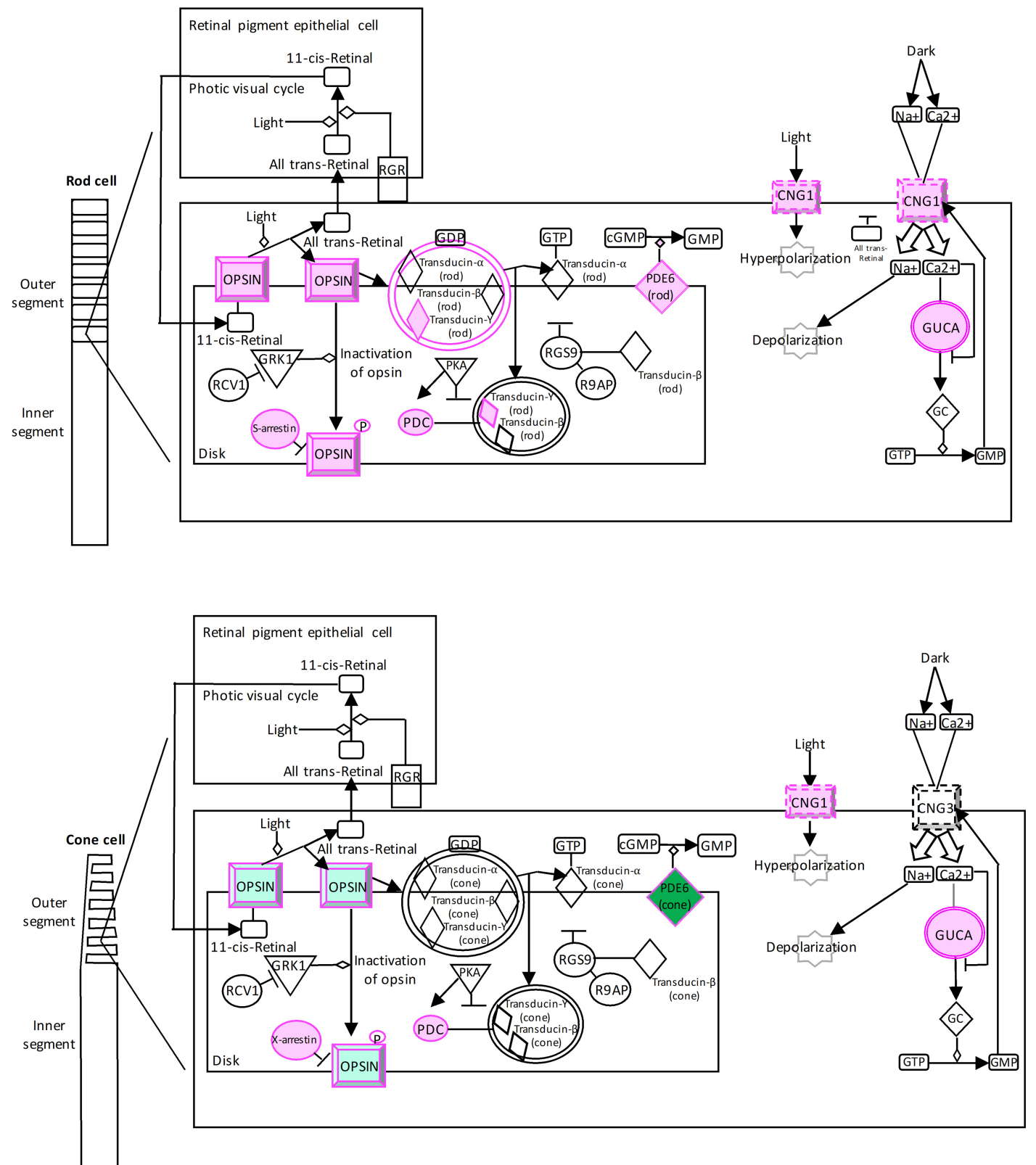

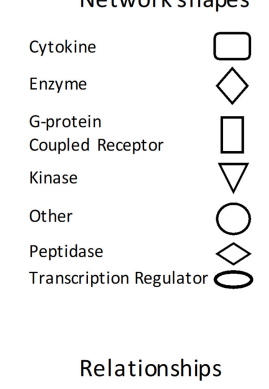

Indirect interaction 

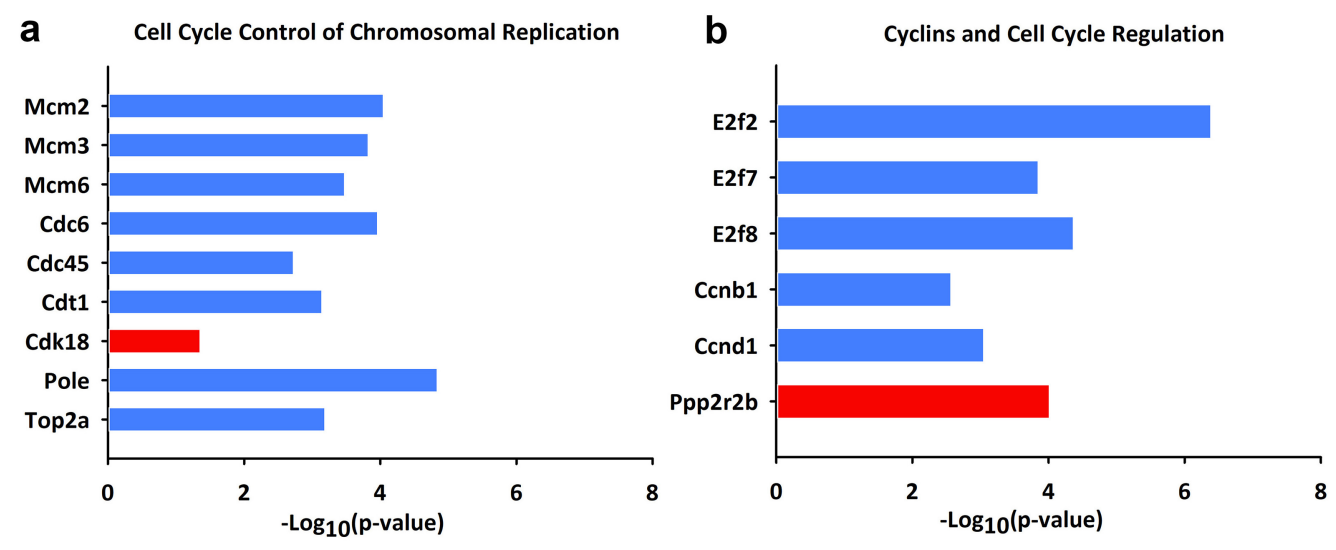

C
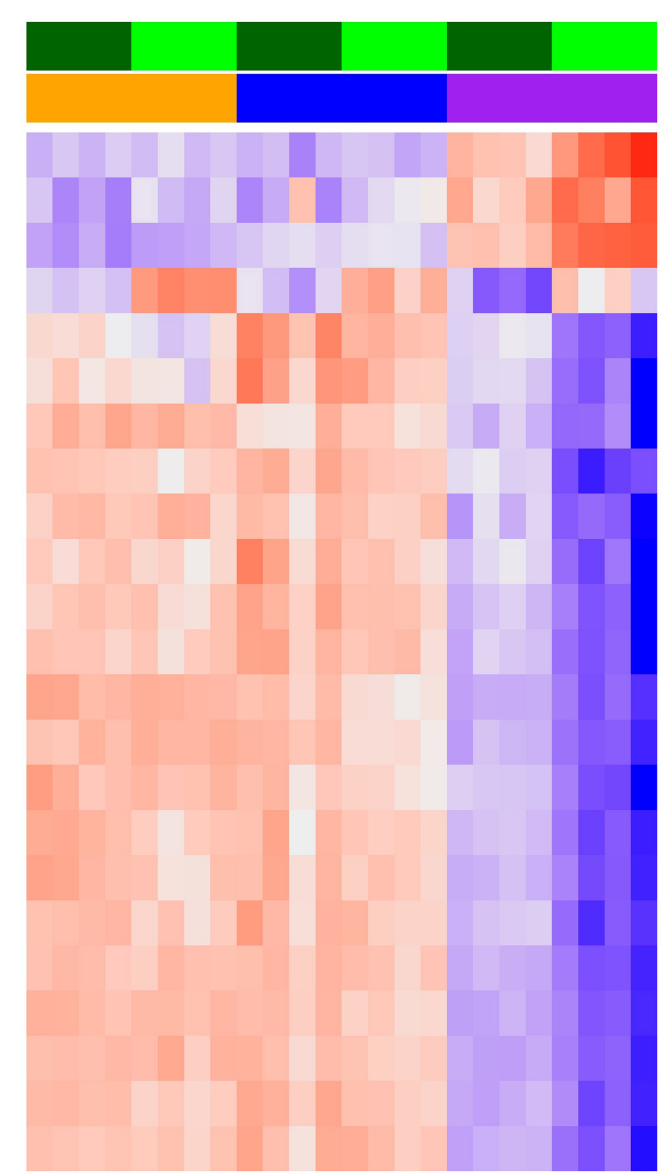

Ppp2r2b

Cdk18

Plk5

Mlh1

E2f8

Cond1

Ccnb1

E2f2

Fanca

Kif11

Bard1

Espl1

Mcm2

Pole

Plk1

E2f7

Cdt1

Brip1

Cdc6

$\mathrm{Mcm} 3$

Cdc45

Mcm6

Top2a 